Pacific Journal of Mathematics

ON THE UNIVERSALITY OF SYSTEMS OF WORDS IN 


\section{ON THE UNIVERSALITY OF SYSTEMS OF WORDS IN PERMUTATION GROUPS}

\section{MaNFred Droste and Saharon ShelaH}

In the classes of infinite symmetric groups, their normal subgroups, and their factor groups, we determine those groups which are equivalent in the sense that they may not be distinguished by the solvability of a system of finitely many equations in variables and parameters.

1. Introduction and results. Recently, several authors $[1,3-5,8$, 9, 12] studied the solvability of equations of the form $w\left(x_{1}, \ldots, x_{n}\right)=y$, where $w$ is a group word, in various kinds of groups. In $[1,3,12]$ this problem was considered for infinite symmetric groups. Here we consider the simultaneous solvability of several equations of a similar form in infinite symmetric groups, their normal subgroups, and their factor-groups.

Let $G$ be a group, $x_{1}, \ldots, x_{n}$ variables, $y_{1}, \ldots, y_{m}$ parameters, and $w_{i}=w_{i}\left(x_{1}, \ldots, x_{n} ; y_{1}, \ldots, y_{m}\right)(i \in I)$ group words in these variables and parameters. We say that $W=\left\{w_{i} \mid i \in I\right\}$ is $G$-universal if $G$ satisfies the following property:

For all $y_{1}, \ldots, y_{m} \in G$ there exist $x_{1}, \ldots, x_{n} \in G$ such that for all $i \in I, w_{i}\left(x_{1}, \ldots, x_{n} ; y_{1}, \ldots, y_{m}\right)=e$.

Two groups $G$ and $H$ will be called equationally equivalent, $G \equiv_{\text {eq }} H$, if for any finite set $W$ of words $w_{i}$ as above, $W$ is $G$-universal iff $W$ is $H$-universal.

Let $S_{\nu}$ denote the infinite symmetric group of all permutations of a set of cardinality $\aleph_{\nu}$ and, for $0 \leq \tau \leq \nu+1, S_{\nu}^{\tau}$ its normal subgroup comprising all permutations moving less than $\aleph_{\tau}$ elements of the underlying set. The problem of the elementary equivalence (definability) of the groups $S_{\nu}(\nu \geq 0)$ was solved in Shelah [11]. Here we will consider the problem of the equational equivalence of the groups $S_{\nu}$. A very similar problem was suggested by J. Isbell, cf. [6; p. 20]. Throughout this paper, let $V=\left\{v_{1}, v_{2}, v_{3}\right\}$ be the following set of words in parameters $y_{1}, y_{2}$ and variables $x_{1}, x_{2}, x_{3}$ :

$$
\begin{aligned}
& v_{i}=y_{i}^{-1} \cdot x_{1}^{-1} \cdot y_{i} \cdot x_{1} \quad(i=1,2), \\
& v_{3}=y_{1}^{-1} \cdot x_{2}^{-1} \cdot x_{1} \cdot x_{2} \cdot x_{3}^{-1} \cdot x_{1} \cdot x_{3} .
\end{aligned}
$$


Note that $v_{i}=e$ means that $x_{1}$ and $y_{i}$ commute $(i=1,2)$, and $v_{3}=e$ means that $y_{1}$ is a product of two conjugates of $x_{1}$. Using a result of Droste and Göbel [2], we will show:

THEOREM 1. (a) Let $\nu \geq 0$. The following are equivalent:

(1) $V$ is $S_{\nu}$-universal.

(2) $V$ is $\left(S_{\nu} / S_{\nu}^{\nu}\right)$-universal.

(3) $\boldsymbol{\aleph}_{\nu}>2^{\aleph_{0}}$.

(b) Let $\mu, \nu \geq 0$ and $\aleph_{\mu}>2^{\aleph_{0}}$. The following are equivalent:

(1) $S_{\nu} \equiv{ }_{\text {eq }} S_{\mu}$.

(2) $S_{\nu} / S_{\nu}^{\nu} \equiv{ }_{\text {eq }} S_{\mu} / S_{\mu}^{\mu}$.

(3) $\aleph_{\nu}>2^{\aleph_{0}}$.

We also obtain the subsequent generalization of Ehrenfeucht et al. [3; Theorem 3] which is partly a consequence of a result of Moran [7]:

THEOREM 2 Let $\nu, \mu \geq 0$. The following are equivalent:

(1) For any finite set $W=\left\{w_{i} \mid i \in I\right\}$ of words $w_{i}=w_{i}\left(x_{1}, \ldots, x_{n} ; y\right)$ in one parameter $y$ and variables $x_{1}, \ldots, x_{n}, W$ is $S_{\nu}$-universal iff $W$ is $S_{\mu}$-universal.

(2) Either (i) $\nu=\mu=0$, or (ii) $\nu, \mu>0$.

In particular, $S_{0} \not \equiv$ eq $S_{\nu}$ whenever $\nu>0$. Hence the following is an immediate consequence of Theorems 1 and 2:

Corollary 3. Assume (CH). Then, for any $\nu, \mu \geq 0$, we have $S_{\nu} \equiv{ }_{\text {eq }} S_{\mu}$ iff either $\nu=\mu=0$ or $\nu=\mu=1$ or $\nu, \mu \geq 2$.

Next we just note the following

THEOREM. It is consistent with (ZFC $+2^{\aleph_{0}}$ arbitrarily large) that: $(*)$ $S_{\nu} \equiv{ }_{\text {eq }} S_{\mu}$ whenever $\aleph_{0}<\aleph_{\nu}<\aleph_{\mu} \leq 2^{\aleph_{0}}$.

This is done by starting with a model $V$ of set theory; then choose $\kappa=\kappa^{\kappa_{0}}$, and force by adding $\kappa$ Cohen reals. As we do not yet know whether $(*)$ is provable in $\mathrm{ZFC}$ or is independent of $\mathrm{ZFC}$, this theorem is of doubtful value at present.

Concerning the permutation groups $S_{\nu}^{\tau}$, we have this result:

THEOREM 4. (a) Let $0 \leq \tau \leq \nu$ and $0 \leq \rho \leq \mu$. Then $S_{\nu}^{\tau} \equiv{ }_{\text {eq }} S_{\mu}^{\rho}$ iff either $\tau=\rho=0$ or $\tau, \rho \geq 1$.

(b) $S_{\nu}^{\nu} \not \equiv{ }_{\text {eq }} S_{\nu}$ whenever $\aleph_{\nu} \leq 2^{\aleph_{0}}$. 
Here, in (b) it remains open whether the assumption $\aleph_{\nu} \leq 2^{\aleph_{0}}$ is necessary.

2. Notation. Let $\mathbf{N}$ denote the set of all positive integers and $\mathbf{N}_{\infty}=\mathbf{N} \cup\left\{\boldsymbol{N}_{0}\right\}$. Let $a^{b}=b^{-1} \cdot a \cdot b$ for $a, b \in G$ (any group), and let $A=\dot{U}_{i \in I} A_{i}$ mean that $A$ is the disjoint union of the $A_{i}$. For a mapping $f$ let $a^{f}$ denote its value at $a$ and $\left.f\right|_{A}$ its restriction to $A$.

$P_{M}$ denotes the group of all permutations of a set $M$ and id ${ }_{M}$ (or id, if there is no ambiguity) the identity map of $M$. Let $p \in P_{M}$. An orbit of $p$ is a minimal $p$-invariant subset of $M$. For any $n \in \mathbf{N}_{\infty}$, let $\bar{p}(n)$ denote the cardinality of the set of all orbits of length $n$ of $p$. Let $\operatorname{supp}(p)=$ $\left\{a \in M \mid a^{p} \neq a\right\}$ denote the support of $p$, and $|p|=|\operatorname{supp}(p)|$. For $\nu \geq 0$ and $0 \leq \tau \leq \nu+1$, let $S_{\nu}=P_{\aleph_{\nu}}, S_{\nu}^{\tau}=\left\{p \in S_{\nu} \| p \mid<\aleph_{\tau}\right\}$ and $A_{\nu}=\left\{p \in S_{\nu}^{0}|p|_{\operatorname{supp}(p)}\right.$ is even $\}$. Then, as is well-known, the groups $A_{\nu}$ and $S_{\nu}^{\tau}(0 \leq \tau \leq \nu)$ are all non-trivial proper normal subgroups of $S_{\nu}$. If $M=\dot{\cup}_{i \in I} M_{i}, p_{i} \in P_{M_{i}}$, and $p \in P_{M}$ such that $\left.p\right|_{M_{i}}=p_{i}$ for each $i \in I$, then we also write $p=\bigoplus_{i \in I} p_{i}$.

If $w\left(x_{1}, \ldots, x_{n} ; y_{1}, \ldots, y_{m}\right)$ is a word in parameters $y_{k}$ and variables $x_{j}$, we also abbreviate it by $w\left(\underset{\sim}{x} ;{\underset{\sim}{k}}_{k}\right)$; we include the indices $j, k$ in this expression in order to indicate that they range over index sets $J, K$ respectively (here $J=\{1, \ldots, n\}, K=\{1, \ldots, m\}$ ).

3. Proof of our results. Before we can prove Theorem 1 , we need a few preparations:

Proposition 3.1. Let $M$ be a set of cardinality $\boldsymbol{\aleph}_{\nu}(\nu \geq 0)$ and $z_{k} \in P_{M}$ for $k \in K$, where $K$ is at most countably-infinite.

(a) If $A \subseteq M$, let $B=\left\{a^{z} \mid a \in A, z \in Z\right\}$ where $Z \subseteq P_{M}$ is the subgroup of $P_{M}$ generated by $\left\{z_{k} \mid k \in K\right\}$. Then $B$ is the smallest subset of $M$ containing $A$ such that $\left.z_{k}\right|_{B} \in P_{B}$ for each $k \in K$. Furthermore, $|B|=|A|$ if $A$ is infinite.

(b) For each cardinality $\boldsymbol{\aleph} \leq \boldsymbol{\aleph}_{\nu}$, there is a decomposition $M=\dot{U}_{l \in L} M_{l}$ such that $z_{k} \mid M_{l} \in P_{M_{l}}$ and $\left|M_{l}\right|=\aleph$ for each $k \in K, l \in L$.

Proof. (a) Obvious.

(b) For $x, y \in M$, let $x \sim y$ if $x=y^{z}$ for some $z \in Z$. This defines an equivalence relation on $M$, and each equivalence class is at most countably-infinite and is invariant under any $z_{k}(k \in K)$. Now choose each $M_{l}(l \in L)$ to be an appropriate union of equivalence classes.

Proposition 3.2. Let $w_{i}=w_{i}\left(\underset{\sim}{\underset{x}{j}} ; y_{k}\right)$ be words, where $i \in I, j \in J$, $k \in K$, and $I, J, K$ are finite, and $\nu \geq \tilde{0}$. If $W=\left\{w_{i} \mid i \in I\right\}$ is $S_{\nu}$-universal, then $W$ is also $S_{\mu}$-universal for any $\mu>\nu$. 
Proof. Let $M$ be a set of cardinality $\boldsymbol{\aleph}_{\mu}$ and $y_{k} \in P_{M}(k \in K)$. By Proposition 3.1(b), split $M=\dot{U}_{l \in L} M_{l}$ such that $\left|M_{l}\right|=\aleph_{\nu}$ and $y_{l, k}=$ $\left.y_{k}\right|_{M_{l}} \in P_{M_{l}}$ for all $k \in K, l \in L$. By assumption, for each $l \in L$ there are $x_{l, j} \in P_{M_{l}}(j \in J)$ such that $w_{i}\left(x_{l, j} ; y_{l, k}\right)=\mathrm{id}_{M_{l}}$ for all $i \in I$. Put $x_{j}=$ $\bigoplus_{l \in L} x_{l, j} \in P_{M}$ for each $j \in J$. Then $w_{i}(\underset{\sim}{x} ; \underbrace{}_{k})=\mathrm{id}{ }_{M}$ for all $i \in I$.

The following lemma is stated in a more general form than actually needed since it may be also of some independent interest. Recall that for a permutation $p$ and $n \in \mathbf{N}_{\infty}, \bar{p}(n)$ denotes the number of orbits of length $n$ of $p$.

LEMMA 3.3. Let $a, b \in S_{0}$ such that a consists of precisely one infinite orbit and $\bar{b}(n) \in \mathbf{N}$ for some $n \in \mathbf{N}$. If $c \in S_{0}$ satisfies $a=a^{c}$ and $b=b^{c}$, then $c=\mathrm{id}$.

Proof. As $P_{\mathbf{Z}} \cong S_{0}$, it suffices to prove this for elements $a, b, c \in S_{\mathbf{Z}}$ where $i^{a}=i+1$ for each $i \in \mathbf{Z}$ and 0 belongs to an orbit of length $n$ of $b$. Let $0^{c}=k \in \mathbf{Z}$. Then $a=a^{c}$ implies $(j \cdot k)^{c}=(j+1) \cdot k$ for any $j \in \mathbf{Z}$. Hence each $j \cdot k(j \in \mathbf{Z})$ belongs to an orbit of length $n$ of $b$. Since $\bar{b}(n)<\infty$, this shows $k=0$. But then $c=$ id by $a=a^{c}$.

Now suppose $\phi: A \rightarrow B$ is a bijection from $A$ onto $B$. Then $\phi$ induces an isomorphism $\psi$ from $P_{A}$ onto $P_{B}$ defined by $b^{p^{\psi}}=b^{\phi^{-1} \cdot p \cdot \phi}$ for each $b \in B, p \in P_{A}$. If $x_{1}, \ldots, x_{n} \in P_{A}, y_{1}, \ldots, y_{n} \in P_{B}(n \in \mathbf{N})$ are such that $x_{i}^{\psi}=y_{i}(i=1, \ldots, n)$, then we also say that $\psi$ is an isomorphism from the algebra $\left\langle A, x_{1}, \ldots, x_{n}\right\rangle$ onto $\left\langle B, y_{1}, \ldots, y_{n}\right\rangle$, induced by $\phi$.

We can now prove Theorems 1 and 2 :

Proof of Theorem 1. (a) (1) $\rightarrow(2): S_{\nu} / S_{\nu}^{\nu}$ is an epimorphic image of $S_{\nu}$.

(2) $\rightarrow$ (3): For contradiction, assume $\aleph_{\nu} \leq 2^{\aleph_{0}}$. Let $M$ be a set of cardinality $\aleph_{\nu}$, and decompose $M=\dot{U}_{j \in J} M_{j}$ such that $\left|M_{j}\right|=\aleph_{0}$ for any $j \in J$, and $|J|=|M|$. For each $j \in J$, choose $y_{j, 1}, y_{j, 2} \in P_{M_{J}}$ such that $y_{j, 1}$ consists of precisely one (infinite) orbit, $\overline{y_{j, 2}}(n) \in \mathbf{N}$ for some $n \in \mathbf{N}$, and whenever $j, k \in J, j \neq k$, then $\overline{y_{j, 2}}(m) \neq \overline{y_{k, 2}}(m)$ for some $m \in \mathbf{N}$. This is possible since $|J| \leq 2^{\aleph_{0}}=\left|S_{0}\right|$. Then put $y_{1}=\bigoplus_{j \in J} y_{j, 1}, y_{2}=$ $\bigoplus_{j \in J} y_{j, 2} \in P_{M}$. Now by (2) there are $x_{i}, z_{i} \in P_{M}$ such that $\left|z_{i}\right|<\boldsymbol{\aleph}_{\nu}$ $(i=1,2,3), y_{i}=y_{i}^{x_{1}} \cdot z_{i}(i=1,2)$, and $y_{1}=x_{1}^{x_{2}} \cdot x_{1}^{x_{3}} \cdot z_{3}$. Let $T$ be the smallest subset of $J$ with $\operatorname{supp}\left(z_{1}\right) \cup \operatorname{supp}\left(z_{2}\right) \subseteq \bigcup_{j \in T} M_{j}^{x_{1}}$. Pick any 
$j \in J \backslash T$. If $N=M_{j}^{x_{1}}$, then $y_{1}=y_{1}^{x_{1}} \cdot z_{1}$ and $\left.z_{1}\right|_{N}=\mathrm{id}_{N}$ imply $\left.y_{1}\right|_{N} \in$ $P_{N}$, and $\left.y_{1}\right|_{N}$ consists of precisely one (infinite) orbit on $N$. Hence $N=M_{k}$ for some $k \in J$. Now $y_{2}=y_{2}^{x_{1}} \cdot z_{2}$ and $\left.z_{2}\right|_{N}=\mathrm{id}_{N}$ yield $\overline{y_{k, 2}}(m)$ $=\overline{y_{j, 2}}(m)$ for any $m \in \mathbf{N}$, thus $k=j$. Hence $M_{j}^{x_{1}}=M_{j}$, implying $\left.x_{1}\right|_{M_{j}}$ $=\mathrm{id}_{M_{j}}$ by Lemma 3.3. This shows $\left|x_{1}\right| \leq|T| \cdot \boldsymbol{\aleph}_{0}<\boldsymbol{\aleph}_{\nu}$ and $\left|y_{1}\right|<\boldsymbol{\aleph}_{\nu}$ by our third equality, an obvious contradiction.

(3) $\rightarrow$ (1): By Proposition 3.2 it suffices to consider the case $\boldsymbol{\aleph}_{\nu}=$ $\left(2^{\aleph_{0}}\right)^{+}$. Let $|M|=\aleph_{\nu}$ and $y_{1}, y_{2} \in P_{M}$. By Proposition 3.1(b), decompose $M=\dot{U}_{j \in J} M_{j}$ with $\left|M_{j}\right|=\boldsymbol{\aleph}_{0}$ and $y_{j, i}=\left.y_{i}\right|_{M_{j}} \in P_{M_{J}}$ for each $j \in J$, $i=1,2$. We call $j, k \in J$ equivalent if there exists an isomorphism from $\left\langle M_{j}, y_{j, 1}, y_{j, 2}\right\rangle$ onto $\left\langle M_{k}, y_{k, 1}, y_{k, 2}\right\rangle$. Since $\left|S_{0}\right|=2^{\aleph_{0}}$, there are at most $2^{\aleph_{0}}$ different equivalence classes on $J$. Hence, by $|J|=\aleph_{\nu}=\left(2^{\aleph_{0}}\right)^{+}$, a regular cardinal, there exists an equivalence class $T \subseteq J$ of cardinality $\boldsymbol{\aleph}_{\nu}$. Now decompose $T=\dot{\cup}_{i \in \mathbf{Z}} T_{i}$ with $\left|T_{i}\right|=|T|$ for each $i \in \mathbf{Z}$, and choose an element $x_{1} \in P_{M}$ such that $\left.x_{1}\right|_{M_{j}}=\mathrm{id}_{M_{j}}$ for each $j \in J \backslash T$, and, for any $i \in \mathbf{Z}, j \in T_{i}$, we have $M_{j}^{x_{1}}=M_{k}$ for some $k \in T_{i+1}$ such that $\left.x_{1}\right|_{M_{j}}$ induces an isomorphism from $\left\langle M_{j}, y_{j, 1}, y_{j, 2}\right\rangle$ onto $\left\langle M_{k}, y_{k, 1}, y_{k, 2}\right\rangle$. Then $y_{i}=y_{i}^{x_{1}}$ for $i=1,2$, and $x_{1}$ consists of $\aleph_{\nu}$ infinite orbits and, possibly, fixed points. Now by Droste and Göbel [2; Theorem 2], any element of $P_{M}$ is a product of two conjugates of $x_{1}$, in particular $y_{1}=x_{1}^{x_{2}} \cdot x_{1}^{x_{3}}$ for some $x_{2}, x_{3} \in P_{M}$. Thus $V$ is $S_{\nu}$-universal.

(b) (1) $\rightarrow$ (3) and (2) $\rightarrow$ (3): By (a) and $\aleph_{\mu}>2^{\aleph_{0}}, V$ is $S_{\mu^{-}}$and $\left(S_{\mu} / S_{\mu}^{\mu}\right)$-universal, hence $S_{\nu}$ and $\left(S_{\nu} / S_{\nu}^{\nu}\right)$-universal by assumption. Now (a) shows that $\aleph_{\nu}>2^{\kappa_{0}}$.

$(3) \rightarrow(1)$ and (3) $\rightarrow(2)$ : Let $w_{i}=w_{i}\left({\underset{\sim}{j}}_{j} ; y_{k}\right)$ be words $(i \in I, j \in J$; $I, J$ finite, $k \in K=\{1, \ldots, n\}$ with $n \in \mathbf{N})$ such that $W=\left\{w_{i} \mid i \in I\right\}$ is $S_{\mu}$-universal $\left(\left(S_{\mu} / S_{\mu}^{\mu}\right)\right.$-universal); we claim that $W$ is $S_{\nu}$-universal $\left(\left(S_{\nu} / S_{\nu}^{\nu}\right)\right.$-universal), respectively. Let $|M|=\aleph_{\nu}$ and $y_{k} \in P_{M}(k \in K)$. By Proposition 3.1(b), there is a decomposition $M=\dot{U}_{l \in L} M_{l}$ such that $\left|M_{l}\right|=\aleph_{0}$ and $y_{l, k}=\left.y_{k}\right|_{M_{l}} \in P_{M_{l}}$ for each $k \in K, l \in L$. We call $l$, $m \in L$ equivalent if there exists an isomorphism from $\left\langle M_{l}, y_{l, 1}, \ldots, y_{l, n}\right\rangle$ onto $\left\langle M_{m}, y_{m, 1}, \ldots, y_{m, n}\right\rangle$.

First let us show that $W$ is $S_{\nu}$-universal. Because of Proposition 3.2, we may assume $\left(2^{\aleph_{0}}\right)^{+}=\boldsymbol{\aleph}_{\nu}<\boldsymbol{\aleph}_{\mu}$. Thus there exists an equivalence class $T \subseteq L$ of cardinality $\boldsymbol{\aleph}_{\nu}$. Fix $t \in T$, choose a set $P$ of cardinality $\boldsymbol{\aleph}_{\mu}$ and, for each $p \in P$, a copy $\left\langle M_{p}, y_{p, 1}, \ldots, y_{p, n}\right\rangle$ of $\left\langle M_{t}, y_{t, 1}, \ldots, y_{t, n}\right\rangle$, and put $A=M \dot{\cup}_{p \in P} M_{p}, y_{k}^{\prime}=y_{k} \oplus \bigoplus_{p \in P} y_{p, k} \in P_{A}(k \in K)$. Since $|A|=\aleph_{\mu}$, by assumption there are $x_{j}^{\prime} \in P_{A}^{p}(j \in J)$ with $w_{i}(\underbrace{\prime}_{j}, \underbrace{\prime}_{k})=\mathrm{id}_{A}$ for all $i \in I$. By Proposition 3.1(a), there is a set $B$ with $M \subseteq B \subseteq A$ and 
$|B|=|M|=\aleph_{\nu}$ such that $y_{k}^{+}=\left.y_{k}^{\prime}\right|_{B}, x_{j}^{+}=\left.x_{j}^{\prime}\right|_{B} \in P_{B}$ for all $k \in K$, $j \in J$; in particular, $w_{i}\left({\underset{\sim}{j}}_{j}^{+} ;{\underset{\sim}{k}}_{k}^{+}\right)=\mathrm{id}_{B}$ for all $i \in I$. Now it is easy to see that $\left\langle M, y_{1}, \ldots, y_{n}\right\rangle$ is isomorphic to $\left\langle B, y_{1}^{+}, \ldots, y_{n}^{+}\right\rangle$. Hence there exist $x_{j} \in P_{M}(j \in J)$ such that $w_{i}\left(\underset{\sim}{x} ; \underline{\sim}_{k}\right)=\mathrm{id}_{M}$ for all $i \in I$, establishing (1).

Next we finish the proof of (2). For any subset $S \subseteq L$, we abbreviate

$$
M_{S}=\bigcup_{l \in S} M_{l} \text { and } y_{k}^{S}=\left.y_{k}\right|_{M_{s}}=\bigoplus_{l \in S} y_{l, k} \in P_{M_{S}} \quad(k \in K) \text {. }
$$

First we claim that for any infinite subset $T \subseteq L$ consisting only of pairwise equivalent elements of $L$ such that $|T|<\boldsymbol{\aleph}_{\mu}$, there are $x_{J}^{T} \in P_{M_{T}}$ $(j \in J)$ with $w_{i}\left({\underset{\sim}{j}}_{j}^{T} ;{\underset{\sim}{k}}_{k}^{T}\right)=\mathrm{id}_{M_{T}}$ for each $i \in I$.

Indeed, fix $t \in \tilde{T}$, choose a set $P$ of cardinality $\boldsymbol{\aleph}_{\mu}$ and, for each $p \in P$, a copy $\left\langle M_{p}, y_{p, 1}, \ldots, y_{p, n}\right\rangle$ of $\left\langle M_{t}, y_{t, 1}, \ldots, y_{t, n}\right\rangle$, and put

$$
A=M_{T} \dot{\cup} \bigcup_{p \in P} M_{p}, y_{k}^{\prime}=y_{k}^{T} \oplus \bigoplus_{p \in P} y_{p, k} \in P_{A} \quad(k \in K) \text {. }
$$

Since $|A|=\aleph_{\mu}$, by assumption there are $x_{j}^{\prime}, z_{i} \in P_{A}(j \in J, i \in I)$ with $\left|z_{i}\right|<\aleph_{\mu}$ and $w_{i}\left({\underset{\sim}{j}}_{j}^{\prime} ; \underline{v}_{k}^{\prime}\right)=z_{i}$ for each $i \in I$. By (3.1), there exists a subset $Q \subseteq P$ of cardinality $|T|$ such that $\left.z_{i}\right|_{M_{Q}}=\mathrm{id}_{M_{Q}},\left.x_{j}^{\prime}\right|_{M_{Q}} \in P_{M_{Q}}$ for each $i \in I, \quad j \in J$. Hence, since $\left\langle M_{Q}, y_{1}^{Q}, \ldots, y_{n}^{Q}\right\rangle$ is isomorphic to $\left\langle M_{T}, y_{1}^{T}, \ldots, y_{n}^{T}\right\rangle$, our claim follows.

Now we define the elements $x_{j} \in P_{M}(j \in J)$ as follows. Whenever $l \in L$ belongs to a finite equivalence class, let $\left.x_{j}\right|_{M_{l}}=\mathrm{id}_{M_{l}}$. For any infinite equivalence class $T \subseteq L$ with $|T|<\aleph_{\mu}$ put $\left.x_{j}\right|_{M_{T}}=x_{j}^{T}$. Finally, if $T \subseteq L$ is an equivalence class with $|T| \geq \boldsymbol{\aleph}_{\mu}$, decompose $T=\dot{\cup}_{t \in T} T_{t}$ with $\left|T_{t}\right|=\boldsymbol{\aleph}_{0}$ for each $t \in T$, and put $\left.x_{j}\right|_{M_{T}}=\oplus_{t \in T} x_{J}^{T_{t}}$. Since there are at most $\left|S_{0}\right|=2^{\boldsymbol{\aleph}_{0}}<\boldsymbol{\aleph}_{\nu}$ equivalence classes on $L$, we obtain $\left|w_{i}\left(\underset{\sim}{x} ; \underset{\sim}{y_{k}}\right)\right|<$ $\aleph_{\nu}$ for each $i \in I$. This shows that $W$ is $\left(S_{\nu} / S_{\nu}^{\nu}\right)$-universal.

Proof of Theorem 2. (1) $\rightarrow$ (2): Put $W=\left\{w_{1}, w_{2}\right\}$ with $w_{1}\left(x_{1}, x_{2} ; y\right)$ $=y^{-1} \cdot x_{2}^{-1} \cdot x_{1} \cdot x_{2} \cdot x_{1}$ and $w_{2}\left(x_{1}, x_{2} ; y\right)=x_{1}^{2}$. Then $W$ is $S_{\nu}$-universal iff every $y \in S_{\nu}$ is a product of two conjugate involutions, i.e. iff $\nu>0$ by Moran [7; Cor. 2.5].

(2) $\rightarrow(1)$ : This can be shown in a similar (but here much easier) vein as the implication $(3) \rightarrow(1)$ of Theorem 1 or almost precisely as in Ehrenfeucht et al. [3; Proof of Theorem 3].

Before we prove Theorem 4, let us note the following useful observation:

REMARK 3.4. Let $w_{i}=w_{i}\left(\underset{\sim}{x} ; y_{k}\right)$ be words, where $i \in I, j \in J, k \in K$, and $I, J, K$ are finite. Let $1 \leq \tau \leq \nu$ and $y_{k} \in S_{\nu}^{\tau}(k \in K)$. If there are $x_{j}^{\prime} \in S_{\nu}(j \in J)$ such that $w_{l}\left({\underset{\sim}{j}}_{j}^{\prime} ; \underline{\sim}_{k}\right)=\mathrm{id}$ for all $i \in I$, then there are also 
$x_{j} \in S_{\nu}^{\tau}(j \in J)$ such that $w_{i}\left(\underset{\sim}{x} ; y_{k}\right)=\mathrm{id}$ for all $i \in I$. In particular, if $W=\left\{w_{i} \mid i \in I\right\}$ is $S_{\nu}$-universal, then $W$ is also $S_{\nu}^{\tau}$-universal.

Proof. Let $M$ be the underlying set of cardinality $\aleph_{\nu}$ and

$$
Y=\bigcup_{k \in K} \operatorname{supp}\left(y_{k}\right)
$$

By Proposition 3.1(a), there is a set $Z \subseteq M$ with $Y \subseteq Z$ and $|Z|<\boldsymbol{\aleph}_{\tau}$ such that $x_{j}^{+}=\left.x_{j}^{\prime}\right|_{Z} \in P_{Z}$ for all $j \in J$. Putting $x_{j}=x_{j}^{+} \oplus \mathrm{id}_{M \backslash Z} \in$ $S_{\nu}^{\tau}(j \in J)$, we obtain $w_{i}\left(\underset{\sim}{x} ; \underset{\sim}{y_{k}}\right)=\mathrm{id}_{M}$ for all $i \in I$.

Proof of Theorem 4. (a) First assume $S_{\nu}^{\tau} \equiv{ }_{\mathrm{eq}} S_{\mu}^{\rho}$. In $S_{\nu}^{0}$ no element of $S_{\nu}^{0} \backslash A_{\nu}$ is a commutator, but if $\rho \geq 1$, each element of $S_{\nu}^{\rho}$ is a commutator in $S_{\nu}^{\rho}$ by Ore [10; pp. 313, 314]. This shows the assertion.

The converse can be established by showing that $S_{\nu}^{\tau} \equiv{ }_{\text {eq }} S_{\nu}^{1}$ whenever $1<\tau \leq \nu$, and that $S_{\nu}^{1} \equiv_{\text {eq }} S_{\mu}^{1}\left(S_{\nu}^{0} \equiv_{\text {eq }} S_{\mu}^{0}\right)$ whenever $\nu, \mu>0(\nu, \mu \geq 0)$. Since the methods applied are similar to (and easier than) the ones used for the proof of the implication $(3) \rightarrow(1)$ of Theorem 1 , we leave the details to the reader.

(b) Clearly, $S_{0}^{0} \not \equiv$ eq $S_{0}$ since any element of $S_{0}$ is a commutator by Ore's Theorem [10]. Hence assume $\nu>0$ now. By Theorem 1, it suffices to show that $V$ is $S_{\nu}^{\nu}$-universal. We present two arguments for this.

Proof I. By Theorem 1, $V$ is $S_{\mu}$-universal for some $\mu>0$ with $\aleph_{\mu}>2^{\aleph_{0}}$. By Remark 3.4, $V$ is $S_{\mu}^{\mu}$-universal. Hence by (a), $V$ is $S_{\nu}^{\nu}$-universal.

Proof II. Let $|M|=\boldsymbol{\aleph}_{\nu}$ and $y_{i} \in P_{M}$ with $\left|y_{i}\right|<\boldsymbol{\aleph}_{\nu}(i=1,2)$. Decompose $M=A \dot{\cup} B$ with $A=Y \dot{\cup} C$ where $Y=\operatorname{supp}\left(y_{1}\right) \cup \operatorname{supp}\left(y_{2}\right)$ and $|C|=|Y|+\aleph_{0}$. Define $x_{1}^{\prime} \in P_{A}$ such that $\left.x_{1}^{\prime}\right|_{Y}=\mathrm{id}_{Y}$ and $\left.x_{1}^{\prime}\right|_{C}$ consists precisely of $|C|$ infinite orbits. By Droste and Göbel [2; Theorem 2], $\left.y_{1}\right|_{A}=x_{1}^{\prime \prime 2} \cdot x_{1}^{\prime x_{3}^{\prime}}$ for some $x_{2}^{\prime}, x_{3}^{\prime} \in P_{A}$. Hence $x_{j}=x_{j}^{\prime} \oplus \mathrm{id}_{B} \in P_{M}$ satisfies $\left|x_{j}\right|<\boldsymbol{\aleph}_{\nu}(j=1,2,3)$ and $v_{i}({\underset{\sim}{j}}_{j} ; \underbrace{}_{k})=\mathrm{id}_{M}$ for $i=1,2,3$.

\section{REFERENCES}

[1] M. Droste, Classes of words universal for the infinite symmetric groups, Algebra Universalis, 20 (1985), 205-216.

[2] M. Droste and R. Göbel, Products of conjugate permutations, Pacific J. Math., 94 (1981), 47-60.

[3] A. Ehrenfeucht, S. Fajtlowicz, J. Malitz, and J. Mycielski, Some problems on the universality of words in groups, Algebra Universalis, 11 (1980), 261-263. 
[4] A. Ehrenfeucht and D. M. Silberger, Universal terms of the form $B^{n} A^{m}$, Algebra Universalis, 10 (1980), 96-116.

[5] R. C. Lyndon, Equations in groups, Bol. Soc. Brasil. Mat., 11 (1980), 79-102.

[6] R. McKenzie, On elementary types of symmetric groups, Algebra Universalis, 1 (1971), 13-20.

[7] G. Moran, The product of two reflection classes of the symmetric group, Discrete Math., 15 (1976), 63-77.

[8] J. Mycielski, Can one solve equations in groups?, Amer. Math. Monthly, 84 (1977), $723-726$.

[9] Equations unsolvable in $\mathrm{GL}_{2}(C)$ and related problems, Amer. Math. Monthly, 85 (1978), 263-265.

[10] O. Ore, Some remarks on commutators, Proc. Amer. Math. Soc., 2 (1951), 307-314.

[11] S. Shelah, First order theory of permutation groups, Israel J. Math., 14 (1973), 149-162; 15 (1973), 437-441.

[12] D. M. Silberger, Are primitive words universal for infinite symmetric groups?, Trans. Amer. Math. Soc., 276 (1983), 841-852.

Received February 25, 1984 and in revised form October 25, 1984. Research by the first author was supported by an award from the Minerva-Stiftung, München. The work was done during a stay of the first-named author at The Hebrew University, Jerusalem, in fall 1982. He would like to thank his colleagues in Jerusalem for their hospitality and a wonderful time.

\section{UNIVERSITÄT ESSEN \\ 4300 ESSEN 1 \\ WEST GERMANY}

AND

THE HEBREW UNIVERSITY

JERUSALEM, ISRAEL 


\section{PACIFIC JOURNAL OF MATHEMATICS EDITORS}

\author{
V. S. VARADARAJAN \\ (Managing Editor) \\ University of California \\ Los Angeles, CA 90024 \\ HeRbert Clemens \\ University of Utah \\ Salt Lake City, UT 84112 \\ R. FINN \\ Stanford University \\ Stanford, CA 94305
}

\author{
HERMANN FLASCHKA \\ University of Arizona \\ Tucson, AZ 85721 \\ RAMESH A. GANGOLLI \\ University of Washington \\ Seattle, WA 98195 \\ VAUGHAN F. R. JONES \\ University of California \\ Berkeley, CA 94720 \\ ROBION KIRBY \\ University of California \\ Berkeley, CA 94720
}

\author{
C. C. MOORE \\ University of California \\ Berkeley, CA 94720 \\ H. SAMELSON \\ Stanford University \\ Stanford, CA 94305 \\ HAROLD STARK \\ University of California, San Diego \\ La Jolla, CA 92093
}

\section{ASSOCIATE EDITORS}
R. Arens
E. F. BECKENBACH
B. H. NEUMANN
F. WOLF
K. YosHIDA (1906-1982)

\section{SUPPORTING INSTITUTIONS}

UNIVERSITY OF ARIZONA
UNIVERSITY OF BRITISH COLUMBIA
CALIFORNIA INSTITUTE OF TECHN
UNIVERSITY OF CALIFORNIA
MONTANA STATE UNIVERSITY
UNIVERSITY OF NEVADA, RENO
NEW MEXICO STATE UNIVERSITY
OREGON STATE UNIVERSITY

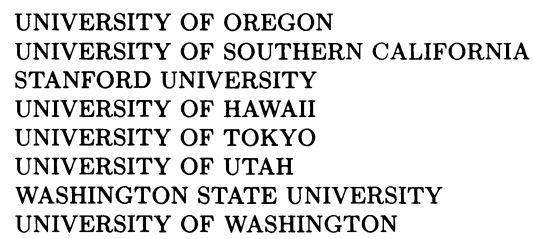

The Supporting Institutions listed above contribute to the cost of publication of this Journal, but they are not owners or publishers and have no responsibility for its content or policies.

Mathematical papers intended for publication in the Pacific Journal of Mathematics should be in typed form or offset-reproduced (not dittoed), double spaced with large margins. Please do not use built up fractions in the text of the manuscript. However, you may use them in the displayed equations. Underline Greek letters in red, German in green, and script in blue. The first paragraph must be capable of being used separately as a synopsis of the entire paper. In particular it should contain no bibliographic references. Please propose a heading for the odd numbered pages of less than 35 characters. Manuscripts, in triplicate, may be sent to any one of the editors. Please classify according to the scheme of Math. Reviews, Index to Vol. 39. Supply name and address of author to whom proofs should be sent. All other communications should be addressed to the managing editor, or Elaine Barth, University of California, Los Angeles, California 90024.

There are page-charges associated with articles appearing in the Pacific Journal of Mathematics. These charges are expected to be paid by the author's University, Government Agency or Company. If the author or authors do not have access to such Institutional support these charges are waived. Single authors will receive 50 free reprints; joint authors will receive a total of 100 free reprints. Additional copies may be obtained at cost in multiples of 50 .

The Pacific Journal of Mathematics is issued monthly as of January 1966. Regular subscription rate: $\$ 190.00$ a year (5 Vols., 10 issues). Special rate: $\$ 95.00$ a year to individual members of supporting institutions.

Subscriptions, orders for numbers issued in the last three calendar years, and changes of address should be sent to Pacific Journal of Mathematics, P.O. Box 969, Carmel Valley, CA 93924, U.S.A. Old back numbers obtainable from Kraus Periodicals Co., Route 100, Millwood, NY 10546.

The Pacific Journal of Mathematics at P.O. Box 969, Carmel Valley, CA 93924 (ISSN 0030-8730) publishes 5 volumes per year. Application to mail at Second-class postage rates is pending at Carmel Valley, California, and additional mailing offices. Postmaster: send address changes to Pacific Journal of Mathematics, P.O. Box 969, Carmel Valley, CA 93924.

PUBLISHED BY PACIFIC JOURNAL OF MATHEMATICS, A NON-PROFIT CORPORATION Copyright (C) 1987 by Pacific Journal of Mathematics 


\section{Pacific Journal of Mathematics \\ Vol. 127, No. $2 \quad$ February, 1987}

Richard Martin Aron and Robert Henry Lohman, A geometric function determined by extreme points of the unit ball of a normed space .... . . 209

Kari Astala and M. S. Ramanujan, $(s)$-nuclear sets and operators $\ldots \ldots \ldots 233$ Earl Robert Berkson, Thomas Alastair Gillespie and Paul Scott Muhly, Analyticity and spectral decompositions of $L^{p}$ for compact abelian

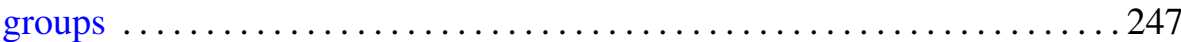

Ronald P. Brown, Real closures of fields at orderings of higher level . . . . . 261

Ronald P. Brown, The behavior of chains of orderings under field

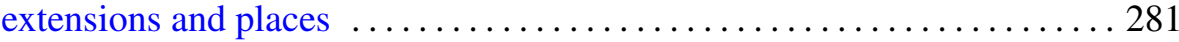

Rodney Graham Downey, Subsets of hypersimple sets .............. 299

Manfred Droste and Saharon Shelah, On the universality of systems of

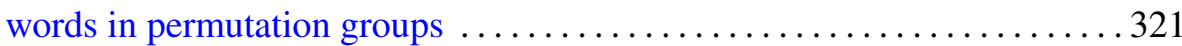

Hidenori Fujiwara, Représentations monomiales des groupes de Lie

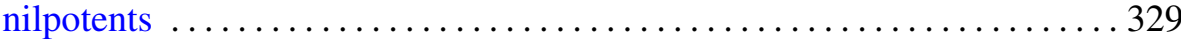

Rupert Lasser, Convolution semigroups on hypergroups $\ldots \ldots \ldots \ldots \ldots 35$

Marcus Marlene Marsh, $u$-mappings on trees $\ldots \ldots \ldots \ldots \ldots \ldots \ldots \ldots \ldots \ldots$

Peter Andrew Symonds, Localization in the classification of flat

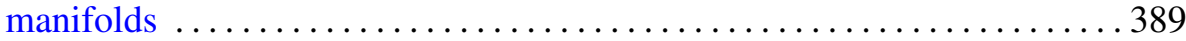

